\title{
SOCKET PRESERVATION- "HEALER OF THE EMPTY SOCKET”- AN OVERVIEW
}

\author{
Dr.Dhwani. K .Dedhia, Dr.Vijayalakshmi.R, Dr.Jaideep Mahendra, Dr. Burnice Nalina Kumari.C \\ Department of Periodontology, Faculty of Dentistry, \\ Meenakshi Academy of Higher Education and Research Institute, Chennai, Tamil Nadu, India.
}

\section{To access \& cite this article}

Website: jidam.idamadras.com

\section{Address for correspondence:}

Dr.Vijayalakshmi.R, MDS

Associate Professor,

Department of Periodontology,

Faculty of Dentistry,

Meenakshi Academy of Higher Education

and Research Institute,

Chennai, Tamil Nadu, India.

Email id: rajaramvijayalakshmi@gmail.com

\section{ABSTRACT}

Extraction is indicated when a tooth cannot be restored or maintained in acceptable conditions for function and/or esthetics. Following tooth extraction, alveolar bone loss and structural and compositional changes of the covering soft tissues, as well as morphological alterations takes place. The repair process results in marked changes in the height and width of the alveolar ridge with an average of $0.7-1.5 \mathrm{~mm}$ of vertical and $4.0-4.5 \mathrm{~mm}$ of horizontal bone resorption which may lead to difficulties at the time of implant placement. This lead to a technique called 'Socket Preservation', also known as alveolar ridge preservation. This procedure reduces bone loss after tooth extraction to preserve the tooth socket in the alveolar bone. The goal of socket preservation is maintenance or enhancement of facial, interproximal gingival contours and height of interproximal papilla. Various materials and procedures are used for socket preservation. Some of them are: connective tissue grafts, free gingival graft, biocol, alloderm, prosthetic "pontic" socket plug, autologous fibrin concentrates, GTR membranes and bone graft materials like autografts, allografts, xenografts and alloplast. Thus, socket preservation at the time of extraction is one of the most significant procedures in the periodontal paradigm for maintenance of health, youth and beauty.

KEYWORDS: Socket preservation, Extraction sockets, Alveolar bone remodeling, GTR, GBR.

$\begin{array}{ll}\text { Received } & : 18.05 .2020 \\ \text { Accepted } & : 05.06 .2020 \\ \text { Published } & : 27.06 .2020\end{array}$




\section{INTRODUCTION:}

The periodontium includes gingiva, connective tissue, cementum, periodontal ligament and alveolar bone. The alveolar bone consists of cortical bone, cancellous trabeculae, and the alveolar bone proper. After the extraction of teeth, there is resorption of the alveolar ridge resulting in its loss of height and width, creating a potential esthetic problem for prosthetic or implant dentistry. The severity of the healing pattern of the extraction socket may pose a problem for the clinician in 2 ways: it creates an esthetic problem in the fabrication of an implant-supported restoration or a conventional prosthesis; and it may make the placement of an implant challenging if not unfeasible. An average of $40 \%-60 \%$ of original height and width is expected to be lost after tooth extraction with the greatest loss happening within the first two years. ${ }^{1}$

\section{What is Socket Preservation?}

The challenge for the dentist is to preserve the quantity and quality of the gingival and osseous tissues lead to the evolution of Socket preservation or alveolar ridge preservation (ARP) (Fig 1). It is a procedure to reduce bone loss after tooth extraction to preserve the dental alveolus in the alveolar bone. It was first described by Greenstein and Ashman and Bruins in 1985. The term socket preservation was first coined by Cohen in 1988 for a procedure designed for prosthetic socket maintenance, ridge preservation and ridge augmentation.

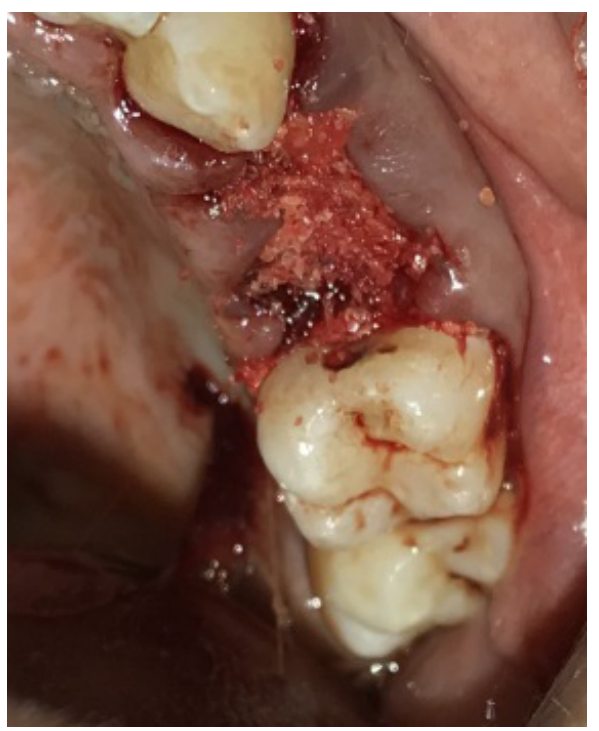

Fig 1: Socket Preservation

\section{CLASSIFICATION:}

\section{According to Elian et $\mathbf{a l}^{2}$ (Fig 2):}

- Type I socket: The facial soft tissue and buccal plate of bone are at normal levels in relation to the cementoenamel junction of the pre-extracted tooth and remain intact post-extraction. Easiest and most predictable to treat.

- Type II socket: Facial soft tissue is present but the buccal plate is partially missing following extraction of the tooth. They are most difficult to diagnose.

- Type III socket: The facial soft tissue and the buccal plate of bone are both markedly reduced after tooth extraction. They are very difficult to treat and require soft tissue augmentation with grafts in a staged approach to rebuild lost tissue.

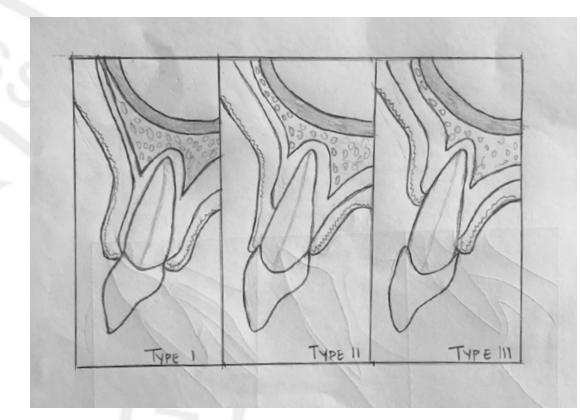

Fig 2: Elian classification of sockets

\section{Chaar et al classification ${ }^{3}$ :}

This classification of extraction sockets focuses on the bone topography of the extraction socket. The protocol for treatment of each socket type takes into account the shape of the remaining bone, the biotype and the location of the socket whether it be in the mandible or maxilla (Box1).

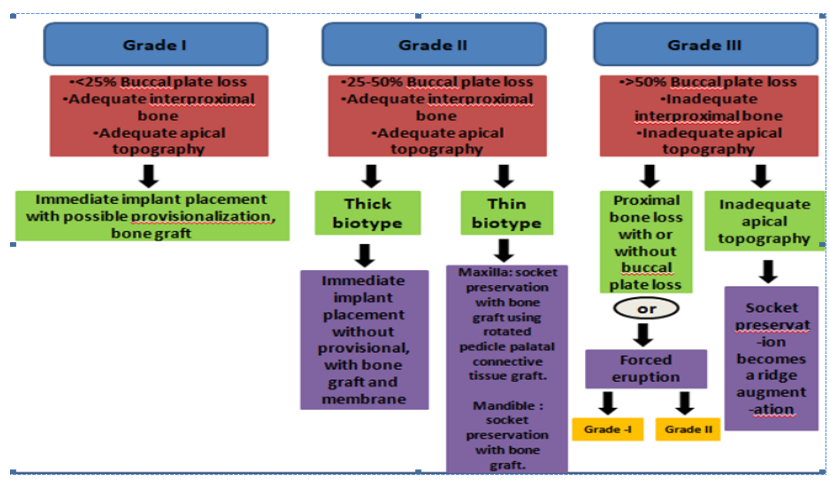

Box 1 : Classification and Treatment Protocol 


\section{Grade I:}

Grade I sockets are most ideal. It had an intact buccal plate, adequate interproximal bone and satisfactory apical topography. There is an intact buccal plate having no fissures or dehiscence and less than $25 \%$ loss of height (Fig 3).

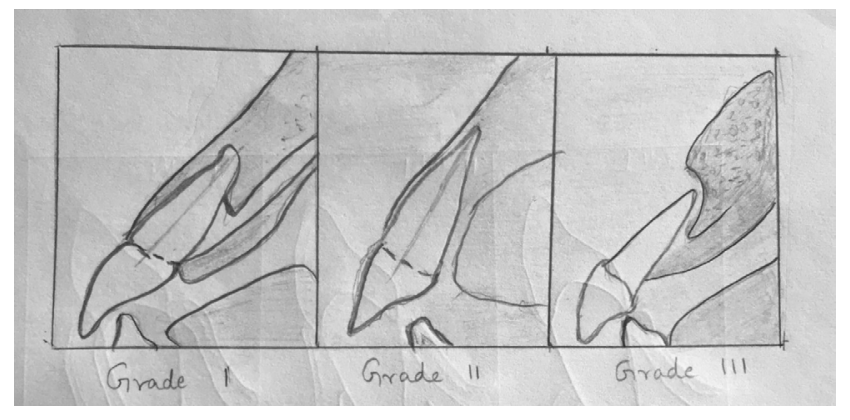

Fig 3: Chaar classification of socket

\section{Grade II:}

Grade II sockets are differentiated from Grade I by the amount and quality of the remaining buccal plate. A grade II socket has a fissure, dehiscence, or deficiency of the buccal plate totalling a $25 \%$ to $50 \%$ loss.

\section{Grade III:}

Grade III sockets are the most deficient and include any socket with inadequate apical topography, insufficient interproximal bone, or more than $50 \%$ loss of buccal plate.

\section{PROCEDURES TO BE OBSERVED TO PRESERVE THE ALVEOLAR SOCKET:}

\section{TOOTH EXTRACTION:}

Atraumatic extraction techniques should be used to minimize damage to the alveolar bone during tooth extraction. The Easy-X-tractor (Fig 4) is a device designed to extract single-rooted teeth with minimal trauma and does not require flap elevation.

If the tooth structure is too broken down, the roots can be carefully sectioned into fragments and extracted without placing pressure on the alveolus. Once the tooth is extracted, the alveolus should be thoroughly debrided, removing all of the granulation tissue.

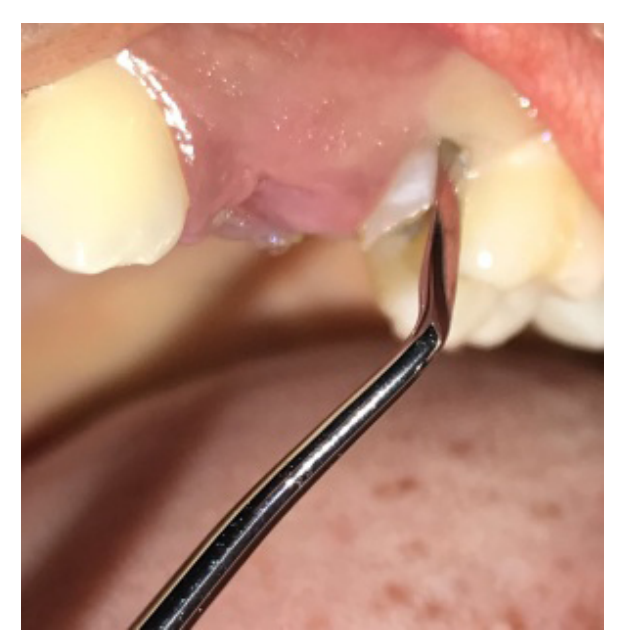

Fig 4: Easy-X-tractor used for extraction

\section{FLAP DESIGN:}

The extraction of a tooth results in a decrease in height of the papillae. The loss of papillae height is increased with the elevation of buccal and lingual flaps. ${ }^{4}$ In areas of esthetic concern, consideration should be given to extracting the tooth and augmenting the socket without elevating a flap or the use of a mini-flap on the buccal side preserving the papillae (Fig 5).

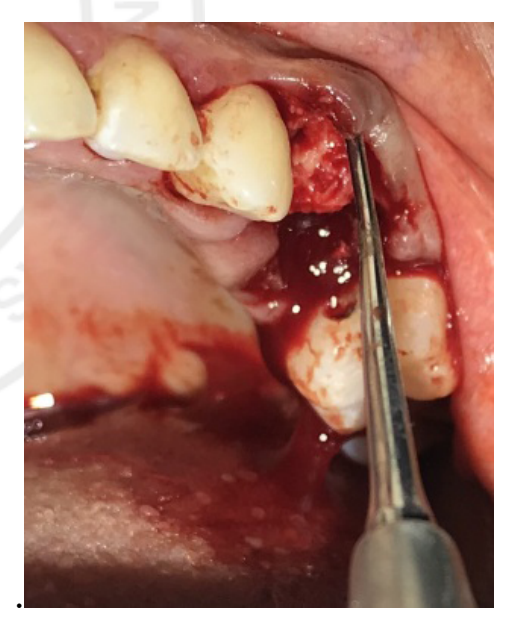

Fig 5: Flap elevation

\section{POST-OPERATIVE MANAGEMENT:}

The most important step in the post-operative management of augmented extraction sockets is the placement and shaping of the temporary tooth that is placed over the extraction area. The loss of papillae height from extraction can be largely restored with the use of removable or fixed provisional appliances. 
HEALING PATTERN OF SOCKET ALVEOLUS:

Residual ridge remodelling, begins with a cascade of inflammatory reactions that is activated immediately after tooth extraction.

Healing of extraction sockets can be explained in five different stages (Fig 6). ${ }^{5}$
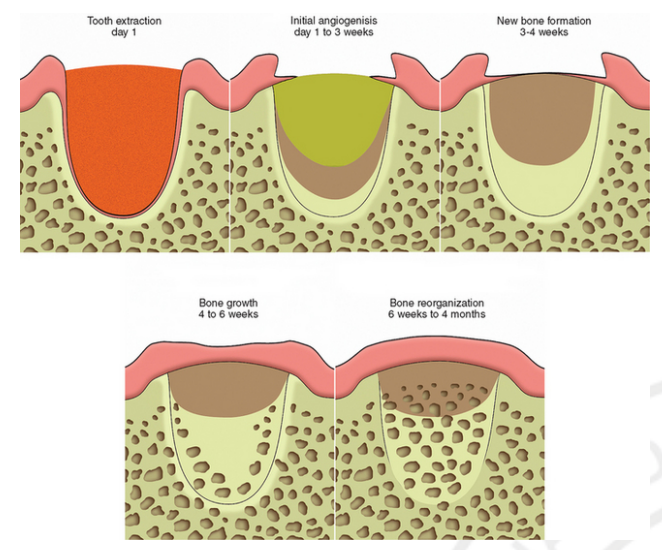

Fig 6: Healing pattern of socket alveolus

\section{GRANULATION STAGE:}

The granulation stage lasts around five days. The early granulation tissue first appears at the bottom of the socket and spreads laterally, up the socket walls.

\section{ANGIOGENIC STAGE:}

The second phase, the initial angiogenic stage, happens within the first week of granulation stage. New trabeculae have begun to appear at the bottom of the socket, and the blood clot in the center has begun to shrink.

\section{NEW BONE FORMATION STAGE:}

The third phase begins two weeks after the tooth extraction. Sinusoid formations that began in the earlier phases yield bone trabeculae. At this point osteoid, can be detected. The bone formation follows the pattern of the sinusoid formations, which have mostly formed vertically starting in the apical region.

\section{BONE GROWTH STAGE:}

The bone growth stage which is characterized by well developed, thickened trabeculae that now fill $2 / 3 \mathrm{rds}$ of the socket. This happens $4-5$ weeks after extraction. This woven bone is referred to as spongiosa. Some sinusoids are still forming in the coronal portion of the socket, while the apical portion appears to be more mature with less sinusoids.

\section{BONE REORGANIZATION STAGE:}

The final stage is the bone reorganization stage which is usually evident around six weeks after extraction. The primary spongiosa now develops into secondary spongiosa, or more lamellar bone, and is present in higher percentages towards the apical end of the residual socket.

\section{TECHNIQUES FOR SOCKET PRESERVATION:}

The techniques available today are based on the principles of guided bone regeneration, which have been used in periodontal regeneration since 1982. ${ }^{6}$ The technique consists of isolating a bony space, in this case an extraction socket with a barrier membrane, to exclude the epithelial cells and thereby have the space filled with bone. The use of a bone replacement graft alone results in some preservation of alveolar height and width but less than with a barrier membrane.

\section{MATERIALS USED FOR SOCKET PRESERVATION:}

Guided Bone Regeneration (GBR) techniques utilize barrier membranes to refrain gingival cells from penetrating into the defect to be regenerated (Fig 7). There are numerous barrier membranes available today for guided bone regeneration.

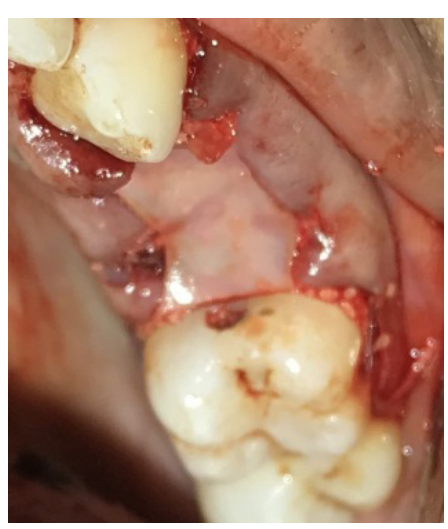

Fig 7: Socket preservation using bone grafts and GTR membrane 


\section{GTR MEMBRANES:}

They can be either resorbable or nonresorbable membranes.

\section{NON RESORBABLE MEMBRANES:}

Non-resorbable membranes were the first devices approved for clinical use. Non-resorbable membranes require a second surgical procedure for removal. The need for additional surgery is accompanied by concerns over patient acceptance, time, cost, and possible morbidity associated with any surgical procedure.

Nyman and colleagues initially used a membrane constructed from Millipore ${ }^{\circledR}$ (cellulose acetate) filters. ${ }^{7}$

Polytetrafluoroethylene is a fluorocarbon polymer with exceptional inertness and biocompatibility.

Teflon ${ }^{\circledR}$, an expanded polytetrafluoroethylene (e-PTFE) membrane consists of 2 parts: a collar portion, having open pores to allow ingrowth of connective tissue and to prevent epithelial migration; and an occlusive portion, preventing the flap tissues from coming into contact with the root surface.

The Gore-Tex expanded polytetrafluoroethylene periodontal device features two structural designs to address specific needs. An open microstructure collar, corresponding to the coronal aspect of the device, to promote connective tissue in-growth and to support wound stability and inhibit epithelial migration. ${ }^{8}$ The remainder of the device is a partially occlusive, structurally relatively stable membrane serving to provide a space for regeneration as well as a barrier towards gingival flap tissue invasion or collapse onto the root surface.

The Gore-Tex expanded polytetrafluoroethylene membrane has been modified by incorporation of titanium reinforcements. The titanium reinforcement is set between two layers of expanded polytetrafluoroethylene, resulting in a device with identical surface properties and improved mechanical strength.

Use of a resin-ionomer barrier has excellent space-making properties; however, it could be difficult to fabricate in-situ.

A rubber dam offers little rigidity to assure space maintenance, can be tedious to manipulate, and exhibits no tissue integration.

A composite non-absorbable device made out of knitted nylon fabric mechanically bonded onto a semipermeable silicone membrane and coated with collagen peptides (Bio Brane) has also been used.

\section{RESORBABLE MEMBRANES:}

Resorbable membranes do not require additional surgery for removal, which reduces patient discomfort, chair-side time and related cost, while eliminating potential surgery-related morbidity.

\section{A. Natural products:}

Collagen is the principal component of connective tissue and provides structural support for tissues throughout the body. It possesses the ability to stimulate platelet attachment and to enhance fibrin linkage, which may assist initial clot formation and stabilization, leading to enhanced regeneration. ${ }^{9}$

A type I collagen guided tissue regeneration membrane derived from bovine deep flexor (Achilles) tendon is widely used.

Another type I collagen membrane derived from calf pericardium and cross-linked by diphenylphosphorylazide has been evaluated for guided tissue regeneration. Other natural products tested as guided tissue regeneration devices include duramater, cargile membrane, oxidized cellulose and laminar bone.

\section{B. Synthetic products:}

Synthetic absorbable devices most commonly used are poly-a-hydroxy acids, which include polylactic acid, polyglycolic acid, and their copolymer, polyglycolide-lactide.

A double-layered absorbable device (GUIDOR) made of polylactic acid and a citric acid ester was the first to gain FDA approval. 
Another synthetic absorbable device (RESOLUT) is a composite consisting of an occlusive membrane of glycolide and lactide copolymer and a porous web structure of bonded polyglycolide fiber. Fiber of polyglactin 910, a copolymer of glycolide and L-lactide (90/10 molar ratio), is used to prepare a tightly woven mesh (VICRYL Periodontal Mesh). The ATRISORB barrier is the only approved guided tissue regeneration device to be manufactured chairside.

Acellular dermal matrix (ADM) is derived from human donor skin tissue. ${ }^{10}$ It is commercially available from tissue banks sanctioned by the American Association of Tissue Banks (AATB).

\section{GUIDED BONE REGENERATION:}

The PASS principle:

P- Primary wound closure

A- Angiogenesis

S- Space maintenance

S- Stabilization

remains a cornerstone for successful guided bone regeneration.

\section{TYPES OF BONE GRAFTS:}

The 4 types of grafts being used are autografts, allografts, xenografts and alloplasts.

\section{AUTOGRAFTS:}

Autografts are taken from one part of a patient's body and transferred to another. The basis for current periodontal bone grafting procedures can be traced to Nabers and O'Leary in 1965.

Osseous coagulum is obtained by using rotary instruments on intra-oral bone in the surgical site and then mixing the particles of bone with the patient's blood.

Bone blend is cortical or cancellous intraoral bone that is obtained with a trephine, chisel or rongeur. It is placed in an amalgam capsule and triturated into particle size in the range of 100 to 200 $\mu \mathrm{m}$.

Autografts can be either extra-oral or intra- oral in origin.

\section{A) EXTRA - ORAL AUTOGRAFT:}

Autografts of iliac cancellous bone and marrow offer the greatest potential for induction of new bone in the periodontium.

Iliac bone marrow autografts (Fig 8) have proven to be the most predictable graft materials for bone growth. However, they are no longer popular. Complications associated with the use of fresh iliac bone marrow include root resorption and ankylosis, in regards to bone grafting around teeth. ${ }^{11}$

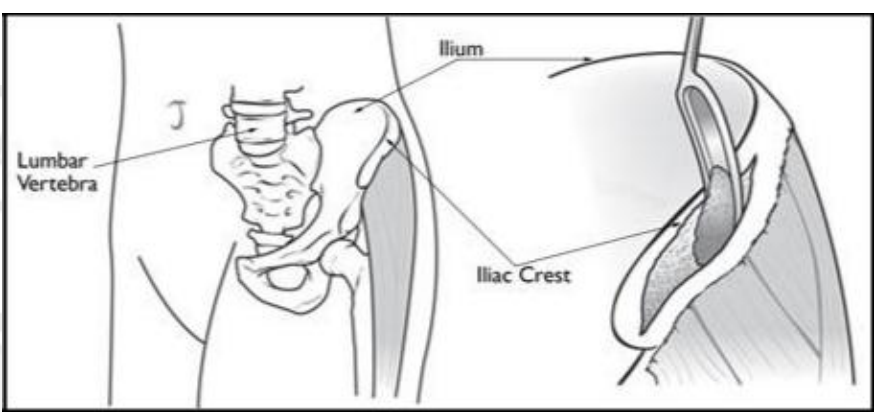

Fig 8: Iliac bone marrow autograft

B) INTRA ORAL AUTOGRAFTS:

Intra oral autografts have been harvested from various intra-oral sites including edentulous ridges, the maxillary tuberosity, post- extraction healing sites and tori or exostoses.

\section{ALLOGRAFTS:}

They consist of tissue transferred from one individual to another genetically dissimilar individual of the same species.

These grafts can be categorized as demineralized frozen or freeze-dried bone allograft (dFDBA) or mineralized frozen or freeze-dried bone allograft (FDBA). FDBA provides an osteoconductive scaffold and elicits slower resorption than dFDBA when implanted in mesenchymal tissues. ${ }^{12}$

\section{XENOGRAFTS :}

They are tissue grafts transferred from one species to a different species. It has been observed in some short-term studies that while the placement 
of biomaterial in alveolar sockets may promote bone formation and ridge preservation, the graft may also delay healing.

\section{ALLOPLASTS:}

Alloplastic bone substitutes like calcium sulfate, bioactive glass and polymers are used. ${ }^{13}$ The Bio-Col technique involves the placement of Deproteinized Bovine Bone Matrix (DBBM) particles in the extraction socket and then covered with a collagen plug or membrane sutured into place.

\section{RECENT TRENDS:}

- Novel tissue engineering therapies have been developed including the delivery of growth factors incorporated in carriers, the stimulation of the selective production of growth factors using gene therapy, and the delivery of expanded cellular constructs.

- The use of rhBMP-2 absorbed in a collagen sponge for alveolar ridge preservation after tooth extraction was published in $1997 .{ }^{14}$

- PDGF-BB in a $\beta$-TCP carrier is a material accepted from the FDA for regeneration of bone and PDL in guided tissue regeneration procedures. ${ }^{15}$

- One of the naturally obtained membrane for socket preservation is platelet-rich fibrin (PRF). Plateletrich fibrin (PRF) is the second generation of platelet concentrates.

- More recently, tissue repair cells (TRC), a cell construct derived from each patient's bone marrow and cultivated using automated bioreactors to concentrations not achievable through a simple bone marrow aspiration, were evaluated in socket healing. ${ }^{16}$

\section{CONCLUSION:}

\section{"The future lies in preserving the past." - Peter Westbrook}

Bone resorption after tooth extraction averages about 3-5 $\mathrm{mm}$ in a period of 6 months. Socket preservation procedures are effective in limiting horizontal and vertical ridge alteration following tooth extraction. The use of barrier membranes alone might improve normal wound healing in extraction sites. The reduction in bone resorption averages upto $2-4 \mathrm{~mm}$ in width of the alveolar bone with the help of socket preservation. Hence this technique along with the help of GTR and GBR is very effective in preserving the alveolar socket.

\section{FINANCIAL SUPPORT AND \\ SPONSORSHIP:}

\section{Nil}

\section{CONFLICTS OF INTEREST:}

There are no conflicts of interest.

\section{REFERENCES:}

1. Caneva M, Botticelli D, Salata LA, SouzaSL, Bressan E, Lang NP. Flap vs flapless surgical approach at immediate implants: A histomorphometric study in dogs. Clin Oral Implants Res 2010;21:1314-1319.

2. Juodzbalys G. Instrument for extraction socket measurement in immediate implant installation. Clin Oral Implants Res 2003;14(2):144-149.

3. El EC, Oshman S, Fallah PA. Singlerooted extraction sockets: Classification and treatment protocol. Compendium of continuing education in dentistry 2016;37(8):537-541.

4. Douglass G. Alveolar ridge preservation at tooth extraction. J Calif Dent Assoc 2005;33:223-231.

5. Ohta Y. Comparative changes in microvasculature and bone during healing of implant and extraction sites. J Oral Implantol 1993;19(3):184-198.

6. Nyman S. New attachment following surgical treatment of human periodontal diseases. J Clin Periodont 1982;9:290-296.

7. Scantlebury TV. A decade of technology development for guided tissue regeneration. J Periodontol 1993;64:1129-1137.

8. Hardwick R, Hayes BK, Flynn C. Devices for dentoalveolar regeneration: An upto date literature review. J Periodontol 1995;66:495505.

9. Nevins ML, Camelo M, Schupbach P, Kim 
DM, Camelo JM and Nevins M. Human histologic evaluation of mineralized collagen bone substitute and recombinant plateletderived growth factor-bb to create bone for implant placement in extraction socket defects at 4 and 6 months: A case series. Int $\mathbf{J}$ Periodont Rest Dent 2009;29:129-139.

10. Luczyszyn SM, Papalexiou V, Novaes AB, Grisi MFM, Souza SLS, Taba M. Acellular dermal matrix and hydroxyapatite in prevention of ridge deformities after tooth extraction. Implant Dent 2005;14(2):176-184.

11. Schallhorn RG. Post-operative problems associated with iliac transplants. J Periodontol 1972;43(1):3-9.

12. Committee on research, science and therapy of the American Academy of Periodontology. Tissue banking of bone allografts used in periodontal regeneration. J Periodontol 2001;72(6):834-838.

13. Froum S, Orlowski W. Ridge preservation utilizing an alloplast prior to implant placement: Clinical and histological case reports. Pract Periodontics Aesthet Dent 2000;12(4):393-402.

14. Cochran DL, Jones AA, Lilly LC, Fiorellini JP and Howell H. Evaluation of recombinant human bone morphogenetic protein-2 in oral applications including the use of endosseous implants: 3-year results of a pilot study in humans. J Periodontol 2000;71:1241-1257.

15. Kaigler D, Pagni G, Park CH, Tarle SA, Bartel RL and Giannobile WV. Angiogenic and osteogenic potential of bone repair cells for craniofacial regeneration. Tissue Eng 2010;16:2809-2820.

16. Zhang Y, Ruan Z, Shen M, Tan L, Huang W, Wang $L$ et al. Clinical effect of platelet-rich fibrin on the preservation of the alveolar ridge following tooth extraction. Exp Ther Med 2018;15(3):2277-2286. 